\title{
Closed Pan-talar Dislocation with Posterior Talar Process Fracture
}

\author{
${ }^{1} \mathrm{M}$ Pramod Kumar, ${ }^{2} \mathrm{KM}$ Gopinath, ${ }^{3} \mathrm{BN}$ Roshan Kumar, ${ }^{4} \mathrm{GA}$ Gautham Balaji
}

\begin{abstract}
Total dislocation of the talus has been reported as $0.06 \%$ of all dislocations and $2 \%$ of all talar fractures. It usually occurs from considerable violence. Total dislocation of the talus is frequently an open injury, or the skin may be tented over the dislocated talus leading to skin slough. Closed total dislocation of talus with posterior process injury is rare. The functional prognosis is poor due to osteonecrosis of the talus which develops in the majority of cases. Review of literature reported successful closed reduction in cases of closed pan-talar dislocation under anesthesia. However, there were few cases where there was difficulty in closed reduction. Operative technique has also been described in different reports of similar cases.
\end{abstract}

Case report: We present a case of pan-talar dislocation of the left talus in a 25-year-old road accident victim, with posterior talar process fracture. Reduction of dislocation was attempted in emergency department by external manipulation. Reduction process failed, and hence planned for reduction under anesthesia. It required a open reduction after a unsuccessful closed reduction attempt. The talus after reduction was found to be unstable hence, stabilized with trans-calcaneotalar Steinmann pin. At 1-year follow-up, the right ankle was pain free and stable. Motion was satisfactory. The talus after a follow-up of 1 year did not show any signs of subluxation or avascular necrosis.

Conclusion: The main obstacle to closed reduction appeared to be talus had button holed through dorsal fascia. The talus after reduction was found to be unstable hence stabilized with trans-calcaneotalar Steinmann pin. The management of the associated fracture will depend on many factors, particularly displacement of the fracture fragments.

Keywords: Closed, Dislocation, Operative treatment, Talus.

How to cite this article: Kumar MP, Gopinath KM, Kumar BNR, Balaji GAG. Closed Pan-talar Dislocation with Posterior Talar Process Fracture. J Med Sci 2015;1(2):32-35.

Source of support: Nil

Conflict of interest: None

\footnotetext{
${ }^{1}$ Assistant Professor, ${ }^{2}$ Associate Professor and Unit Head ${ }^{3}$ Professor and Head, ${ }^{4}$ Postgraduate

${ }^{1-4}$ Department of Orthopedics, RajaRajeswari Medical College and Hospital, Bengaluru, Karnataka, India
}

Corresponding Author: GA Gautham Balaji, Postgraduate Department of Orthopedics, RajaRajeswari Medical College and Hospital, Bengaluru, Karnataka, India, Phone: 9738220940 e-mail: drgauthambalaji@yahoo.co.in

\section{INTRODUCTION}

A closed total dislocation of the talus from all its surrounding joints (talonavicular, tibiotalar, subtalar) not accompanied by a fracture (talus, navicular, calcaneus, malleoli) is an extremely rare injury caused by a high-energy trauma. Its exact incidence is unknown. Closed total talus dislocations are rare. ${ }^{1}$ No standard treatment protocol exists regarding treatment of these rare traumatic injuries. ${ }^{2}$ Avascular necrosis is a wellknown, severe complication postfracture or subluxation of the talus. Type and localization of injury often permit conclusions regarding the probability of bone necrosis. ${ }^{3}$

Reviews of case reports open total talar dislocations show subsequent development of infection and avascular necrosis of the talar dome, and poor clinical and functional outcomes associated with this injury. Open total talar dislocation is a relatively rare but debilitating injury. Techniques for managing such injuries include reimplantation of an extruded talus, open reduction internal fixation and fusion (immediate or delayed)., ${ }^{4,5}$

Literature cites infection and avascular necrosis as the main complications associated with a talar extrusion. Good open fracture protocol can reduce the risk of infection. Reduction of the extruded talus is preferable to preserve function and maintain normal hindfoot anatomy. Talectomy should be reserved as a salvage procedure. $^{6}$

Avascular necrosis is dependant on the amount of blood supply that remains to a bone which is notorious for its problematic vascularity. ${ }^{7}$ We present our method of treatment in this case with closed total talus dislocation without fracture.

\section{CASE REPORT}

A 25-year-old male, with alleged history of fall from a two wheeler, presented in our emergency with pain, swelling and deformity of his left ankle.

\section{EXAMINATION}

- Swelling over the left foot and ankle and varus deformity of the forefoot (Fig. 1)

- On palpation, bony swelling was present below the lateral malleolus with hollowness below the medial malleolus 
- The dorsalis pedis and posterior tibial pulsations were palpated as normal

- Movement of ankle was painful and tender

- No external injury

- No associated injury

- No hypoesthesia over the foot or ankle

- There was no sign of hyperlaxity of other joints.

\section{INVESTIGATIONS}

\section{Plain Radiography}

Talus was found completely dislocated from the tibiotalar, talocalcaneal and talonavicular joints. The talus lay horizontally just below the lateral malleolus (Figs 2A and B).

\section{Computerized Tomography}

Showed pan-talar dislocation with fracture of the posterior process of the talus (Fig. 3).

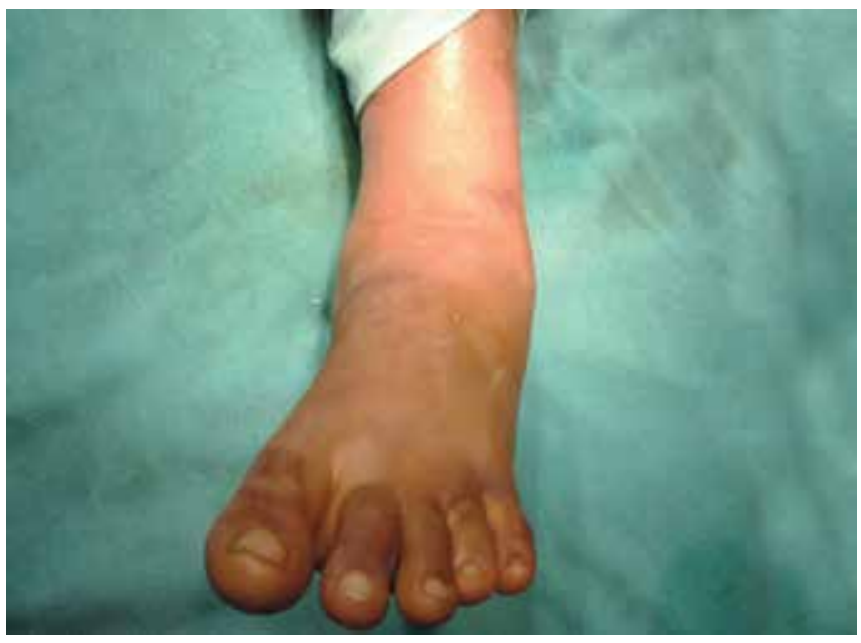

Fig. 1: Swelling over the left foot and ankle, and varus deformity of the forefoot

\section{Emergency Room}

Gentle manipulation was tried twice in the emergency department with the foot in plantar flexion, pronation of foot with knee flexion. Reduction process failed, and hence planned for reduction under anesthesia (Figs $4 \mathrm{~A}$ and B).

\section{Operative Procedure in the Operation Theater}

- Under general anesthesia, reduction was done which also failed.

- Open reduction was done through medial approach. Medial malleolus osteotomy done to approach the talus.

- Open reduction was successful and revealed that the talus had 'button-holed' through the dorsal fascia preventing closed reduction.

- Joint was thoroughly washed.

- Ankle joint was found to be unstable plantigrade, hence a $3.5 \mathrm{~mm}$ Steinmann pin passed retrograde from calcaneum to the tibia under fluoroscopy.

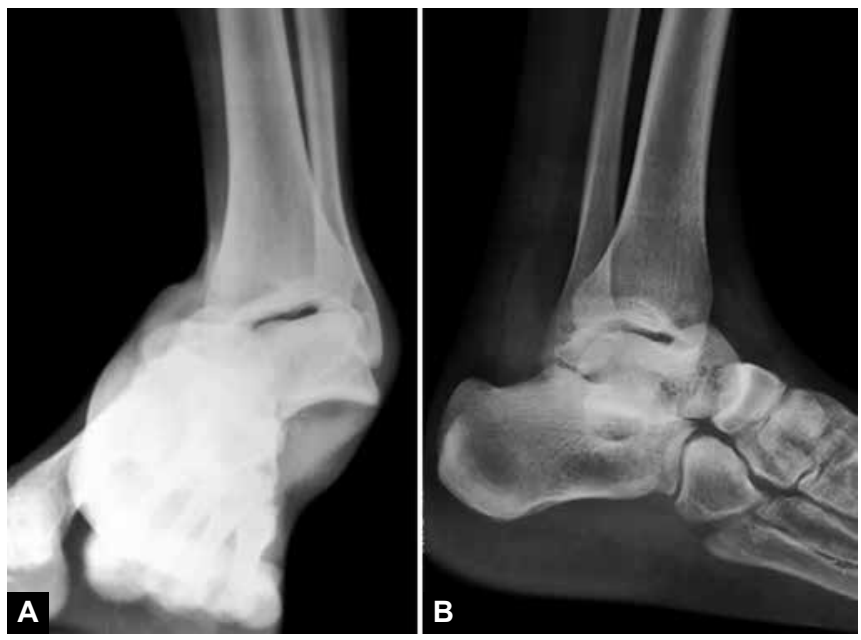

Figs 2A and B: Anteroposterior and lateral radiographs of total lateral talar dislocation
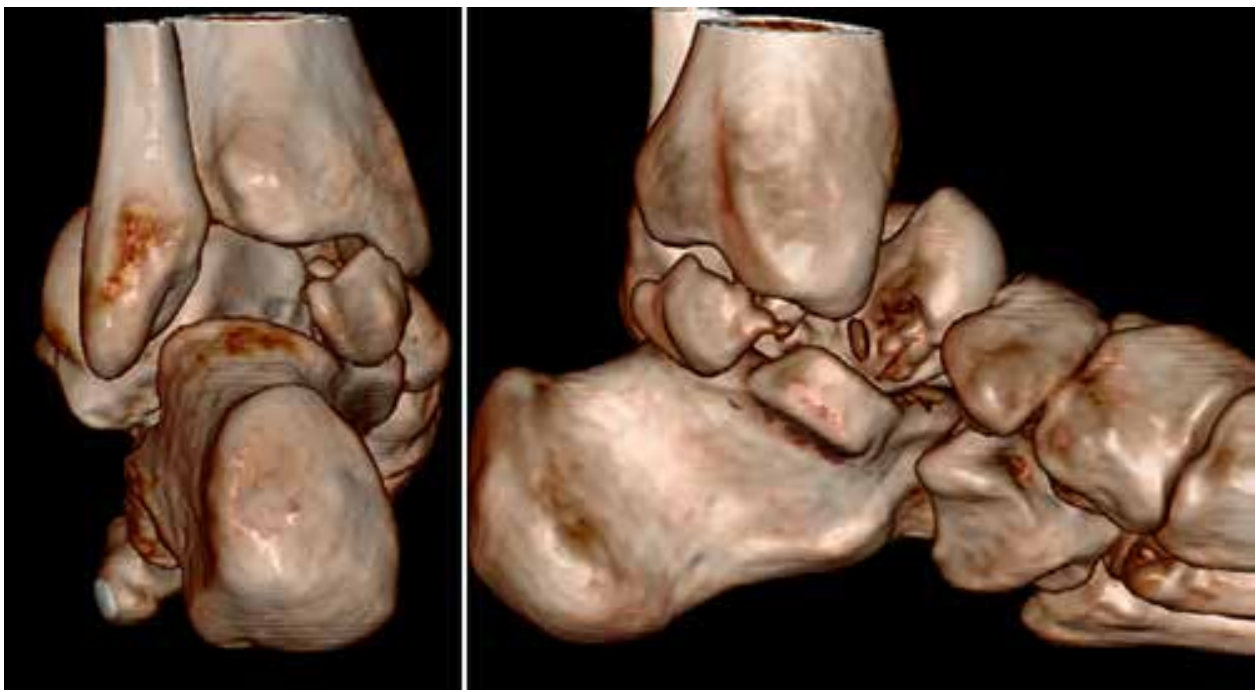

Fig. 3: Computerized tomography (CT) showed pan-talar dislocation with fracture of the posterior process of the talus 


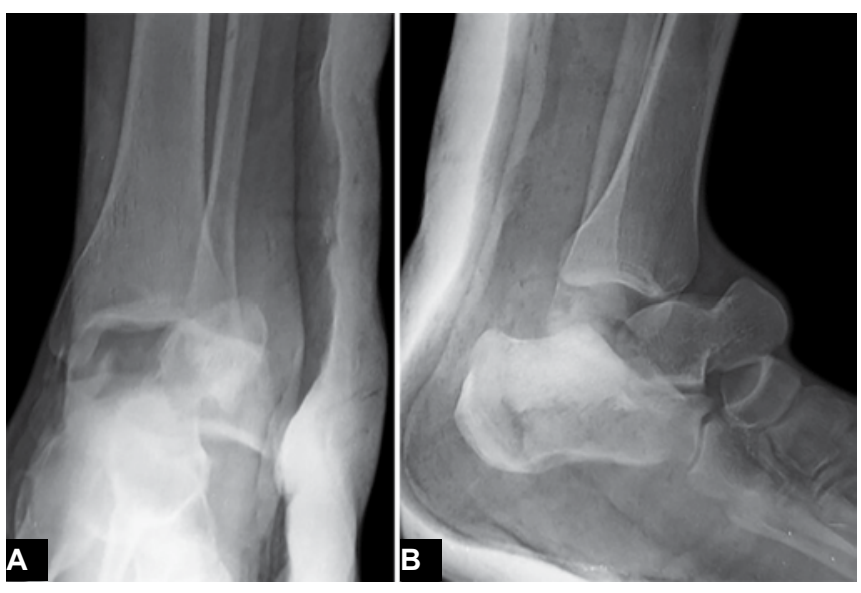

Figs 4A and B: Anteroposterior and lateral views after closed reduction
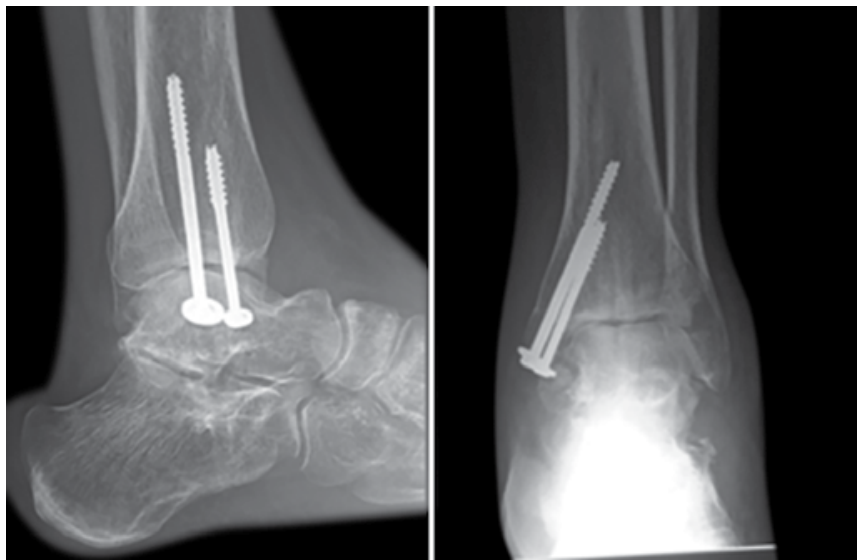

Fig. 6: Anteroposterior and lateral views of ankle after the Steinmann pin was removed

- Medial malleolus was fixed with two cannulated cancellous screws. Comminuted posterior process was removed (Fig. 5).

- Postoperatively, immobilization was done with a below knee slab.

\section{Postoperative}

- After removing the sutures, a nonweight-bearing below knee cast was applied for 6 weeks.

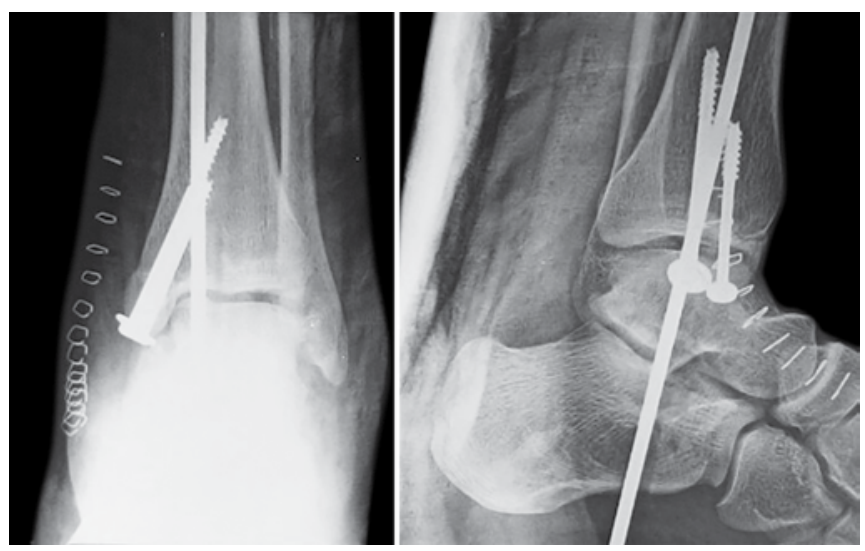

Fig. 5: Postoperative lateral X-rays showing the reduced ankle with Steinmann pin and medial malleolus with $4 \mathrm{~mm}$ cannulated cancellous screws

- After 6 weeks, the Steinmann pin was removed (Fig. 6).

- Left ankle was kept in nonweight-bearing with gradual mobilization for another 6 weeks.

\section{Follow-up}

- Patient was reviewed after 6 months and was found to have pain free $10^{\circ}$ dorsiflexion and $40^{\circ}$ plantar flexion.

- Radiographic examination showed no sign of avascular necrosis of the talus (Figs 7A and B).

\section{DISCUSSION}

Total dislocation of the talus has been reported as $0.06 \%$ of all dislocations and $2 \%$ of all talar fractures. From previous studies, incidence of open pan-talar dislocation was between 10 and $40 \%$. In closed dislocations, significant injury of the soft tissue is present, especially of the skin tented over the head of the talus. ${ }^{8}$

Zimmer and Johnson summarized eight major series plus their experience and reported 79.5\% medial, $17 \%$ lateral, $2.5 \%$ posterior and $1 \%$ anterior dislocations. Associated fractures were more frequently seen in lateral dislocations. They reported $25 \%$ open dislocations and 4 to $5 \%$ avascular necrosis. ${ }^{9}$

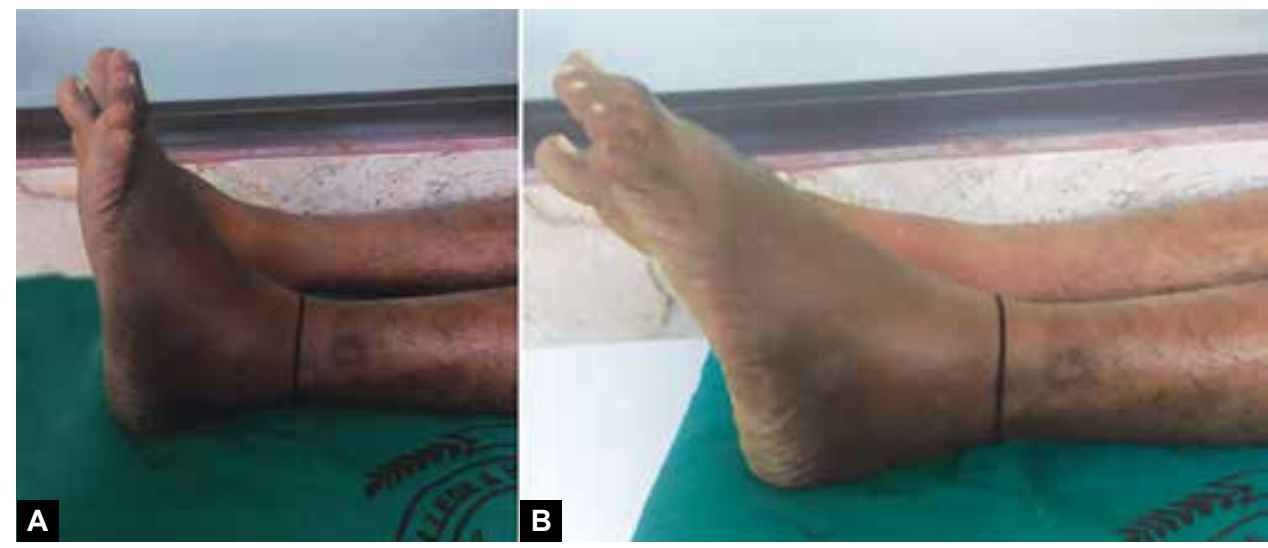

Figs $7 \mathrm{~A}$ and $\mathrm{B}$ : (A) $10^{\circ}$ dorsiflexion and (B) $40^{\circ}$ plantar flexion at 6 months 
DeLee and Curtis reported the results of 17 patients involving $70 \%$ medial, $23 \%$ lateral and $5 \%$ anterior dislocations. Open dislocations were reported in $17 \%$ and associated fractures involving talocalcaneal and talonavicular joints in $47 \%{ }^{10}$

The difficulty in closed reduction that we faced in the present case was reported in many previous studies. In our case, closed reduction was not achieved as there the talus had 'button-holed' through the dorsal fascia preventing reduction. The operative technique discussed in this case was partly described in different reports of similar cases.

\section{CONCLUSION}

Total dislocation of talus with posterior process injury is a rare injury, usually an open injury. But, we report a case of closed total dislocation of talus with posterior process fracture, which required a open reduction after a unsuccessful closed reduction attempt. The main obstacle to closed reduction appeared to be talus which had gone through a button hole in the dorsal fascia. The talus after reduction was found to be unstable, hence, stabilized with Steinmann pin. The management of the associated fracture will depend on many factors, particularly displacement of the fracture fragments.

\section{REFERENCES}

1. El Ibrahimi A, Shimi M, Elidrissi M, Daodi A, Elmrini A. A case of closed total dislocation of talus and literature review. Am J Emerg Med 2011;29(4):475. e1-e3.

2. Heylen S, De Baets T, Verstraete P. Closed total talus dislocation: a case report. Acta Orthop Belg 2011;77(6):838-842.

3. Schiffer G, Jubel A, Elsner A, Andermahr J. Complete talar dislocation without late osteonecrosis: clinical case and anatomic study. J Foot Ankle Surg 2007;46(2):120-123.

4. Ely EE, Konstantakos EK, Laughlin RT, Johnson RM, Binski JC. Total dislocation of the talus and the navicular: a case report. J Orthop Trauma 2009;23(7):546-549.

5. Burston JL, Isenegger P, Zellweger R. Open total talus dislocation: clinical and functional outcomes: a case series. J Trauma 2011;68(6):1453-1458.

6. Fleming J, Hurley KK. Total talar extrusion: a case report. J Foot Ankle Surg 2009;48(6):690. e19-e23.

7. Xarchas KC, Psillakis IG, Kazakos KJ, Pelekas S, Ververidis AN, Verettas DA. Total dislocation of the talus without a fracture open or closed treatment? Report of two cases and review of the literature. Open Orthop J 2009;3:52-55.

8. Weston JT, Liu X, Wandtke ME, Liu J, Ebraheim NE. A systematic review of total dislocation of the talus. Orthop Surg 2015;7:97-101.

9. Fotiadis E, Lyrtzis C, Svarnas T, Koimtzis M, Akritopoulou K, Chalidis B. Closed subtalar dislocation with non-displaced fractures of talus and navicular: a case report and review of the literature. Cases J 2009;2:8793.

10. DeLee JC, Curtis R. Subtalar dislocation of the foot. J Bone and Joint Surg 1982 Mar 1;64(3):433-437. 